\title{
A Hardware Model of the Human Cerebral Cortex: Basic Principles and Prospects
}

\author{
Andrey Molyakov* \\ Institute of information technologies and cybersecurity, Russia
}

*Corresponding author: Andrey Molyakov, Institute of information technologies and cybersecurity, Russian State University for the Humanities, Russia

\begin{tabular}{|c|c|}
\hline ARTICLE INFO & ABSTRACT \\
\hline Received: 彗 January 21, 2020 & $\begin{array}{l}\text { Keywords: Neuronal Model; Non-Von Neumann Architecture; Organic Memristors; } \\
\text { Massive Multi-Thread Processor }\end{array}$ \\
\hline
\end{tabular}

Citation: Andrey Molyakov. A Hardware

Model of the Human Cerebral Cortex: Basic Principles and Prospects. Biomed J Sci \& Tech Res 25(1)-2020. BJSTR. MS.ID.004158.

\section{Mini Review}

The time has come for computing inspired by the structure of the brain. Algorithms that use neural networks and deep learning, imitating some aspects of the human brain, allow digital computers to reach incredible heights in language translation, finding elusive patterns in huge volumes of data. But while engineers continue to actively develop this computational strategy, capable of much, the energy efficiency of digital computing is reaching its limit. Our data centers and supercomputers already consume megawatts - $2 \%$ of all electricity consumed in the USA goes to data centers. And the human brain is well worth 20 watts, and this is a small fraction of the energy contained in the daily food consumed. If we want to improve computing systems, we need to make computers look like brains [1,2]. A surge of interest in neuromorphic technologies is associated with this idea, promising to move computers beyond simple neural networks, towards circuits that work as neurons and synapses. The development of physical circuits similar to the brain is already quite well developed. The work done in my laboratory and other institutions around the world over the past 35 years has led to the creation of artificial nerve components, similar to synapses and dendrites, which react and generate electrical signals in much the same way as real ones.

And yet the fact that we can come up with such a system, says that we did not have long before the advent of smaller-scale chips suitable for use in portable and wearable electronics. Such gadgets will consume little energy, so a neuromorphic chip with high energy efficiency - even if it takes on only part of the calculations, say, signal processing - can become revolutionary. Existing features, such as speech recognition, can work in noisy environments. You can even imagine the smartphones of the future carrying out real-time speech translation in a conversation between two people. Think about this: over the 40 years since the advent of integrated circuits for signal processing, Moore's law has improved their energy efficiency by about 1,000 times. Very brain-like neuromorphic chips can easily surpass these improvements, reducing energy consumption by another 100 million times. As a result, calculations that previously needed a data center fit in the palm of your hand. In an ideal machine that approaches the brain, you will need to recreate analogues of all the main functional components of the brain: synapses that connect neurons and allow them to receive and respond to signals; dendrites combining and conducting local calculations based on incoming signals; the nucleus, or soma, is the region of each neutron, combining the input from dendrites and transmitting the output to the axon [3].

The simplest versions of these basic components are already implemented in silicon. This work began with the same metal oxide semiconductor, or MOSFET, whose billions of copies are used to build logic circuits in modern digital processors. These devices have a lot in common with neurons [4]. Neurons work by means of voltage- 
controlled barriers, and their electrical and chemical activity depends mainly on the channels in which ions move between the inner and outer spaces of the cell. This is a smooth, analog process in which there is a constant accumulation or reduction of the signal, instead of simple on / off operations. MOSFETs are also voltage controlled and operate by the movements of individual charge units. And when the MOSFETs operate in the "subthreshold" mode, without reaching the voltage threshold, switching the on and off modes, the amount of current flowing through the device is very small - less than one thousandth of the current that can be found in typical switches or digital gates.

\section{New Principles of Developing Synthetic Neuronal Network}

The idea that the physics of subthreshold transistors can be used to create brain-like circuits was expressed by Carver Mead from Caltech, who contributed to the revolution in the field of superlarge integrated circuits in the 1970s. Mil pointed out that chip designers did not use many interesting aspects of their behavior, using transistors exclusively for digital logic. This process, as he wrote in 1990, is similar to the fact that "all the beautiful physics that exist in transistors are crushed to zeros and ones, and then the AND and OR gates are painfully built on this basis to reinvent the multiplication." A more "physical" or physics-based computer could do more calculations per unit of energy than a conventional digital one. Mead predicted that such a computer would take up less space. In subsequent years, engineers of neuromorphic systems created all the basic blocks of the brain from silicon with high biological accuracy.

Dendrites, axon, and neuron soma can be made from standard transistors and other elements. For example, in 2005, Ethan Farquhar and I created a neural circuit from a set of six MOSFETs and a bunch of capacitors. Our model emitted electrically impulses, very similar to those that emit catfish neuron squid - a longstanding object of experiments. Moreover, our scheme achieved such indicators with current and energy consumption levels close to those existing in the squid brain. If we wanted to use analog circuits to model the equations derived by neuroscientists to describe this behavior, we would have to use 10 times more transistors. Performing such calculations on a digital computer would require even more space. Synapses and catfish: a floating-gate transistor capable of storing various amounts of charge can be used to create a coordinate array of artificial synapses (bottom left). Electronic versions of other components of a neuron, such as a catfish, can be made from standard transistors and other components [5].

Synapses are a little harder to emulate. A device behaving like a synapse should be able to remember what state it is in, respond in a certain way to the incoming signal, and adapt its responses over time. There are several potential approaches to creating synapses. The most developed of them is a Single-Transistor Learning Synapse (STLS) synapse, which my colleagues in Kaltech and I worked on in the 1990s, when I was a graduate student at Mead. We first introduced STLS in 1994, and it has become an important tool for engineers creating modern analog circuits - for example, physical neural networks. In neural networks, each node in the network has a weight associated with it, and these weights determine how data from different nodes is combined. STLS was the first device capable of containing a set of different weights and reprogramming on the fly. In addition, the device is non-volatile, that is, it remembers its state, even when not in use - this circumstance significantly reduces the need for energy.

STLS is a type of floating gate transistor, a device used to create cells in flash memory. In a conventional MOSFET, the gate controls the current passing through the channel. The floating gate transistor has a second gate, between the electric gate and the channel. This shutter is not directly connected to earth or any other component. Thanks to this electrical insulation, reinforced with high-quality silicon insulators, the charge is stored in a floating gate for a long time. This shutter is able to take a different amount of charge, and therefore can give an electrical response at many levels - and this is necessary to create an artificial synapse that can vary its response to the stimulus. My colleagues and I used STLS to demonstrate the first coordinate network, a computational model that is popular with nanodevice researchers. In a two-dimensional array, devices are located at the intersection of input lines going from top to bottom and output lines going from left to right. Such a configuration is useful in that it allows you to program the connecting force of each "synapse" separately, without interfering with other elements of the array.

Thanks, in particular to the recent DARPA program called Synapse, in the field of engineering neuromorphing there has been a surge in research on artificial synapses created from nanodevices such as memristors, resistive memory and phase-state memory, as well as floating-gate devices. But these new artificial synapses will be hard to improve based on arrays with a floating shutter twenty years ago. Memristors and other types of new memory are difficult to program. The architecture of some of them is such that it is quite difficult to access a specific device in a coordinate array. Others require a dedicated transistor for programming, which significantly increases their size. Since the memory with a floating shutter can be programmed for a wide range of values, it is easier to adjust to compensate for production deviations from device to device compared to other nanodevices. Several research groups that studied neuromorphic devices tried to incorporate nanodevices into their designs and as a result began to use devices with a floating shutter. And how do we combine all these brainlike components? In the human brain, neurons and synapses are intertwined. Developers of neuromorphic chips must also choose an integrated approach with the placement of all components on one chip. But in many laboratories, you will not find this: to make it easier to work with research projects, separate base units are in different places. 
Synapses can be placed in an array outside the chip. Connections can go through another chip Brain memory elements, such as synapse power, are mixed with signal-transmitting components. And the "wires" of the brain - dendrites and axons that transmit incoming signals and outgoing impulses - are usually short in comparison with the size of the brain, and they do not need a lot of energy to maintain the signal. From anatomy, we know that more than $90 \%$ of neurons connect to only 1,000 neighboring ones. Another big question for the creators of brain-like chips and computers is the algorithms that will have to work on them. Even a slightly brainlike system can give a big advantage over a conventional digital one. For example, in 2004, my group used floating-gate devices to perform multiplication in signal processing, and it took 1,000 times less energy and 100 times less space than a digital system. Over the years, researchers have successfully demonstrated neuromorphic approaches to other types of computing for signal processing. But the brain is still 100,000 times more efficient than these systems. This is because although our current neuromorphic technologies take advantage of the neuron-like physics of transistors, they don't use algorithms like the ones that the brain uses to do its job.

\section{Conclusion}

Today we are just starting to discover these physical algorithms - processes that can enable brain-like chips to work with efficiency close to brain. Four years ago, my group used silicon catfish, synapses, and dendrites to run a word-searching algorithm that recognizes words in audio recordings. This algorithm showed a thousand-fold improvement in energy efficiency compared to analog signal processing. As a result, by reducing the voltage applied to the chips and using smaller transistors, researchers must create chips comparable in performance to the brain in many types of computing. When I started research on neuromorphism, everyone believed that the development of systems similar to the brain would provide us with amazing opportunities. Indeed, now entire industries are built around AI and in-depth learning, and these applications promise to completely transform our mobile devices, financial institutions and the interaction of people in public places. And yet, these applications rely very little on our knowledge of brain function. Over the next 30 years, we will no doubt be able to see how this knowledge is increasingly used. We already have many basic hardware blocks needed to convert neurobiology into a computer. But we need to understand even better how this equipment should work - and which computational schemes will give the best results.

\section{References}

1. Hammarlund P, Ekeberg O (1998) Large neural network simulations on multiple hardware platforms. Journal of Computational Neuroscience 5(4): 443-459.

2. Shahaf G, Eytan D, Gal A, Kermany E, Lyakhov V, et al. (2008) Orderbased representation in random networks of cortical neurons. PLoS computational biology 4(11): e1000228.

3. Bakkum DJ, Guy Ben Ary, Phil Gamblen, Thomas B De Marse, Steve M Potter, et al. (2004) Removing some "A" from AI: Embodied Cultured Networks. Microscope pp: 130-145.

4. Abeles M (1991) Corticonics: Neural Circuits of the Cerebral Cortex. Cambridge University Press P: 296.

5. Edelman GM (1993) Neural Darwinism: selection and reentrant signaling in higher brain function. Neuron 10(2): 115-125.
ISSN: 2574-1241

DOI: $10.26717 /$ BJSTR.2020.25.004158

Andrey Molyakov. Biomed J Sci \& Tech Res

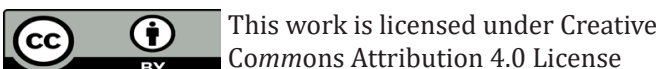

Submission Link: https://biomedres.us/submit-manuscript.php

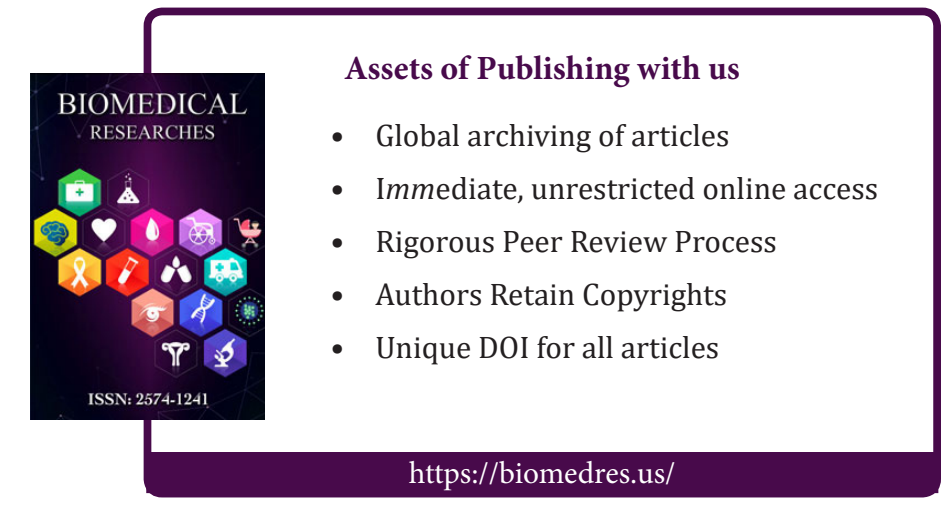

